\title{
COVID-19: disease pathways and gene expression changes predict methylprednisolone can improve out- come in severe cases
}

Sorin Draghici ( $\sim$ sorin@wayne.edu )

Wayne State University

Tuan-Minh Nguyen

Wayne State University

Larry A. Sonna

Bon Air Consulting

Cordelia Ziraldo

Advaita Bioinformatics

Radu Vanciu

Advaita Bioinformatics

Raef Fadel

Henry Ford Health System

Austin Morrison

Henry Ford Health System

Rachel M. Kenney

Henry Ford Health System

George Alangaden

Henry Ford Health System

Mayur Ramesh

Henry Ford Health System

Gil Mor ( $\sim$ gmor@med.wayne.edu )

Wayne State University

\section{Research Article}

Keywords: SARS-CoV2, influenza A, respiratory syncytial virus, hyper-inflammation, drug repurposing, methylprednisolone, gene expression

Posted Date: May 15th, 2020

DOI: https://doi.org/10.21203/rs.3.rs-29392/v1 
License: (c) (i) This work is licensed under a Creative Commons Attribution 4.0 International License. Read Full License 


\section{Abstract}

Current management efforts of COVID-19 include: early diagnosis, use of antivirals, and immune modulation. After the initial viral phase of the illness, identification of the patients developing cytokine storm syndrome is critical.1, 2 Treatment of hyper- inflammation in these patients using existing, approved therapies with proven safety profiles could address the immediate need to reduce the rising mortality. 3 The identification of existing drugs that could modulate the immune response is an immediate need. Here we show that an analysis of the changes in the gene expression, path- ways and putative mechanisms between SARS-CoV2, influenza A, and respiratory syncytial virus can be used to identify FDA-approved drugs that could be repurposed to help COVID-19 patients with severe symptoms related to hyper-inflammation. An important finding is that drugs in the same class may not achieve similar effects. An independent clinical study evaluated 213 subjects, 81 (38\%) and 132 (62\%) in pre-and post-methylprednisolone groups, respectively. Thirty-day all-cause mortality occurred at a significantly lower rate in the post-methylprednisolone group compared to pre-methylprednisolone group $(29.6 \% \mathrm{vs}$. $16.6 \%, p=0.027)$. Clinical results con- firmed the in silico prediction that methylprednisolone could improve outcomes in severe cases of COVID-19. These findings are important for any future pandemic regardless of the virus.

\section{Background}

Most current efforts related to COVID-19 span a number of areas as follows: i) antivi- rals, ii) vaccine development, iii) diagnostic tests, and iv) patient-supporting interven- tions. Without reducing the significance and impact of any of the areas above, there is an important aspect that has not been elucidated: the identification and treatment of patients developing critical conditions and risk of mortality. Mehta et al. state in a very recent Lancet paper 1: "Accumulating evidence suggests that a subgroup of patients with severe COVID-19 might have a cytokine storm syndrome." This cytokine syn- drome correlates with high mortality. We propose that identification and appropriate management of the patients developing cytokine storm syndrome is critical for suc- cessful outcomes. Treatment of hyperinflammation in these patients using existing, approved therapies with proven safety profiles could address the immediate need to reduce the rising mortality.

Unlike other efforts related to COVID-19, the work presented here focuses on: i) understanding immunological response by lung epithelial cells to COVID-19 infec-tion, and ii) identifying drugs that would mitigate or alleviate some of the devastating over-reactions of the host's immune system (e.g. cytokine storm) that lead to poor outcomes, including death.

A SARS-CoV2-specific vaccine or a SARS-CoV2-specific antiviral will reduce the impact of this particular virus in future seasons. However, better understanding the acute reaction of the immune systems and having more tools to mitigate and/or avoid a cytokine storm will be important for any future pandemic regardless of the virus. 


\section{Results}

We used available transcriptomic data to compare A549 lung cell line infected with SARS-CoV-2 vs. mock infection (henceforth A549CoV2vsControl), A549 infected with seasonal influenza A virus vs mock infection (A549IAVvsControl), and A549 in- fected with human respiratory syncytial virus vs mock infection (A549RSVvsControl). We also compared the transcriptional response in primary human bronchial epithelial (NHBE) between cells infected with SARS-CoV2 and mock infection (NHBECoV2vsControl). Finally, we compared the transcriptional response in COVID-19 lung tissues vs. healthy lung tissue (COVID19vsHealthy). These data were collected at Mount Sinai and are available in GEO as the GSE147507 data set 4.

Disrupted genes and biological processes. Fig. S3 shows a comparison of the affected biological processes in COVID19vsHealthy, NHBECoV2vsControl, A549CoV2vsControl, A549IAVvsControl, and A549RSVvsControl. The biological pro- cesses (BPs) are shown ordered by their significance in COVID19vsHealthy. In spite of a larger number of DEGs in the COVID-19-infected lung (815), there are only 7 significant biological processes involved, which may indicate a more coordinated, systemic response. In contrast, the changes in the NHBE cells are characterized by fewer DEGs (only 223) but span more uncoordinated biological processes. This is illustrated in Fig. S1 which shows the BPs ordered in the order of significance from NHBECoV2vsControl. Fig. S2 shows the Venn diagram comparing the differentially expressed genes (DEGs) in the two contrasts. A comparison of the DE genes across the five contrasts is shown in Fig. S4.

Putative mechanisms of disease. We performed an analysis aiming to identify putative mechanisms of disease. As part of this analysis we identified four genes that were predicted to be activated upstream regulators based on the observed changes in their downstream genes. These were IRF9, STAT2, IFNG, and IFNB1. These suggest two different potential mechanisms. The first appears to be triggered by STAT2 and IRF9, which have 16 common target genes that are also all significantly up-regulated (IFI6, IFIT1, IFIT2, IFIT3, IFITM1, IFITM3, OAS1, OAS3, OAS2, MX1, MX2, RSAD2, OASL, XAF1, IRF2, IRF7). This mechanism is also known to be involved in the response to influenza A (see influenza A pathway in Fig. S5).

The second putative mechanism involves interferon beta and gamma, which are targeting 5 common downstream genes: CXCL10, ID01, DOX58, STAT1, which are up-regulated, and HMOX2 which is downregulated. Interferon regulatory factors (IRFs) are subdivided into the interferonic IRFs (IRF2-3-7 and 9), the stress respon- sive IRFs (IRF1 and 5), the hematopoietic IRFs (IRF4 and 8) and morphogenic IRF6. IRF9 is a regulator of type I IFN signaling and is known to interact with STAT2 5 and STAT1 to form the heterotrimeric transcription factor complex (ISGF3) that binds to interferon-stimulated response elements (ISREs) to induce the expression of in- terferon stimulated genes (ISG). During viral infections, ISGs perform two key func- tions: 1) directly limit viral replication by shutting down protein synthesis and triggering apoptosis; 2) ISGs activate key components of the innate and adaptive immune sys- tem, including antigen presentation and production of cytokines. The genes triggered by the STAT2 and IRF9 
pathway include genes responsible for limiting viral replication (IFI6, IFIT1, IFIT2, IFIT3, IFITM1, IFITM3, OAS1, OAS3, OAS2, MX1, MX2, RSAD2, OASL) and inducers of apoptosis (XAF1, IRF2, IRF7). CXCL10, ID01, DOX58, and STAT1 are genes associated with lymphocyte recruitment and immune regulation.

Interestingly, STAT2 and IRF9 together are also identified as activated upstream regulators due to 15 downstream targets even in the NHBECoV2vsControl (see Fig. S6). However, in the NHBE cells, the interferon activators were replaced by an interleukin- based mechanism centered around IL1B, IL6, IL17A, adiponectin (ADIPOQ) and tu- mor necrosis factor (TNF).

We also looked at genes that are known to modulate or inhibit the inflammatory response such as IL1RN IL10, and IL13. In the COVID19vsHealthy, IL1RN was up with a log2 fold change of 6.2 fold (a 78-fold increase, FDR-corrected $p=10^{-6}$ ), IL10 was up 2.8 fold (FDR-corrected $p=0.55$ ), while the measurement for IL13 was not available. In the NHBECoV2vsControl, IL1RN was up only 1.26 fold (FRD-corrected $p=$ 0.035), while measurements for IL10 and IL13 were not available. However, in this contrast, 14 out 15 DE genes immediately downstream of IL 10 and usually in- hibited by IL10 were up-regulated which strongly supports the hypothesis that IL10 is inhibited (FDR-corrected $p=5.17^{-9}$ ).

Impacted pathways. The significantly impacted pathways are shown in Fig. S7 ordered by their significance in COVID19vsHealthy. The p-values represent a com-bination of enrichment and perturbation p-values (see 6 for details) corrected with FDR. Fig. S8 shows the signaling pathways in all 5 experiments, ordered by their significance in NHBECoV2vsControl.

Fig. S9 shows the most impacted pathway, the Cytokine-cytokine interactions. Fig. S10 shows the Chemokine signaling pathway. On this pathway, the impact is due both to the large number of DE genes (26 out of 130), as well as to the clear signal propagation from the chemokines outside the cell (11 chemokines up-regulated), through the chemokine receptor and via the JAK and STAT mechanism. Fig. S12 shows another view of the mechanism involving the genes on this pathway and all their known interactions.

Proposed drugs. Once we identified the main regulatory pathways potentially associated with hyperinflammation we evaluated in silico FDA-approved drugs that could show activity on multiple components of inflammation and consequently could be used for the management of severe COVID-19 cases. We considered the number of DE genes that would be reverted by each drug, as well as calculated a Bonferroni- corrected p-value indicating the suitability of each drug for repurposing in COVID-19 based on two different approaches (see methods). We looked for drugs that have both small p-values as well as revert a larger number of DE genes. The top three drugs drug identified by our analysis are shown in Fig. 1. Methylprednisolone is the drug that was identified as the most likely to work. This drug targets 27 genes that are found to be DE in COVID19vsHealthy. Out of these 27 genes, the drug would revert the changes in 25 of them. The drug also had an extremely significant $p$-value $\left(p=5.72^{-10}\right)$ even after a Bonferroni correction which is the most stringent correction available. Methylprednisolone also reverted 22 out of 22 genes found to be DE in NHBECoV2vsControl, and 25 out of 26 genes found to be DE in 
A549CoV2vsControl. Fig. 2 shows the putative mechanism through methylprednisolone acts on the DE genes in COVID19vsHealthy, and how these genes influence the BPs found to be significantly impacted.

Clinical validation. In an independent study, 213 eligible subjects were en- rolled, 81 (38\%) and 132 (62\%) in pre-and post-methylprednisolone groups, respec- tively. The clinical characteristics and treatments received by the patients are shown in Table S1 and Table S2, respectively. As shown in Table S3 thirty day all-cause mortality occurred at a significantly lower rate in post-methylprednisolone group com- pared to pre-methylprednisolone group $(29.6 \%$ vs. $16.6 \%, p=0.027)$. No statis- tical difference was detected in the proportion of patients prescribed empiric an- tibiotics or the time to empiric therapy. The survival curves of the pre- and post- methylprednisolone groups are shown in Fig. 3.

\section{Discussion}

Two recent papers stress the importance of a clinical phenotyping that would dis- tinguish the phase where the viral pathogenicity is dominant versus when the host inflammatory response overtakes the pathology 2, 3. A strong argument in favor of also targeting the host response is offered by the data on influenza. Even though in- fluenza patients receive optimal anti-viral therapy, approximately $25 \%$ of the critically ill influenza patients still die 3,7 . This suggests that anti-virals alone will not be suffi- cient for COVID-19 either, and the host response to the virus still needs to be taken into consideration.

However, approaches aiming at modulating the immune response face some concerns. In particular, it may seem counter-intuitive to try to diminish the immune response in a patient whose immune system is fighting against a virus. Modulating the immune system is likely unnecessary and counter-productive for patients whose immune system is doing a good job at resolving the infection, while it could potentially be life-saving for those whose inflammatory response has become dysregulated. If a patient has developed severe respiratory symptoms and is hypoxic, the host response that lead to ARDS, sepsis, and organ failure has already been initiated 1. At this point, the focus should shift to supporting the patient's systems and preventing collapse triggered by hyper-inflammation 3 .

An important finding is that drugs in the same class will not have similar effects. For instance, methylprednisolone and prednisolone were predicted to be effective in reverting many of the changes triggered by COVID-19, while other closely-related steroids such as prednisone or dexamethasone were not. Methylprednisolone and prednisolone are steroids currently used to modulate the immune response in rheumatoid arthritis. The putative mechanisms through which these drugs would re- vert the genes dysregulated in COVID-19 are shown in Fig. 4. We also looked at other steroids such as prednisone, dexamethasone, and hydrocortisone. However, prednisone was found to target only 3 genes that are DE in the COVID19vsHealthy and only 2 of the genes that are DE in the NHBECoV2vsControl. From those, pred- nisone would revert only 1 of the $3 \mathrm{DE}$ genes in the COVID19vsHealthy and 0 out of the 2 in the NHBECoV2vsControl. Both yielded insignificant $p$-values ( $p=1$ after Bonferroni) suggesting that prednisone is not expected to be an effective treatment. Prednisolone, dexamethasone, and hydrocortisone belong to the same family of cor- ticosteroidal anti-inflammatory agents and there is also 
a structural similarity between them (see Fig. S14). In spite of this structural similarity, hydrocortisone would only revert 8 out of 10 genes found to be DE in the COVID19vsHealthy ( $p=0.57$ after FDR) and 5 out of 8 genes found to be DE in the NHBECoV2vsControl ( $p=0.038$ after FDR, $p=1$ after Bonferroni). Dexamethasone was found to revert 33 out of 69 of the genes found to be DE in the COVID19vsHealthy ( $p=1$ after FDR correction) and 27 out of 45 genes in the NHBECoV2vsControl ( $p=0.002$ after FDR correction, $p=0.066$ after Bonferroni correction). In short, neither dexamethasone nor hydrocortisone appears to be effective in the COVID-19 lung tissue, although hydrocortisone appears to be marginally effective in the NHBE.

The host inflammatory response in the lungs may lead to acute lung injury and acute respiratory distress syndrome (ARDS). This constitutes the main rationale for potentially using corticosteroids. However, corticosteroids may have adverse effects, an increased risk of secondary infection and delayed viral clearance. A recent article in Lancet reports that clinical evidence does not support corticosteroid treatment for COVID-19 8. However, this report looks at steroids as an entire class of drugs. A re- cent retrospective study of 201 patients with COVID-19 in China found that treatment with methylprednisolone for those who developed ARDS was associated effective in decreasing the risk of death. Among patients with ARDS, treatment with methylpred- nisolone decreased the risk of death ( $\mathrm{HR}, 0.38 ; 95 \% \mathrm{Cl}, 0.20-0.72)$. (23/50 [46\%] with methylprednisolone vs 21/34 9. Both reports are entirely consistent with our findings: corticosteroids in general are NOT expected to help as a class of drugs. However, methylprednisolone and prednisolone are targeting a large number of the genes affected by COVID-19 and are expected to work significantly better than other corticosteroids.

We also looked at other drugs that have already been proposed as repurpos- ing candidates for COVID-19 including: chloroquine, hydroxychloroquine, ery-thromycin, prednisone, dexamethasone, ibuprofen, ritonavir, aspirin, and clopi- dogrel. None of these was predicted to be effective in reverting SARS-CoV2 gene expression changes (see Supplementary Materials).

\section{Methods}

Clinical Validation. We evaluated the methylprednisolone protocol with a single pre- test, single post-test quasi-experiment from March 12-March 27, 2020 at a 5 hospital health system in Michigan. Patients were compared before and after implementation of the methylprednisolone protocol on March 20th. The clinical characteristics of the patients are shown in Table S1. The primary endpoint was 30 day all-cause mortality.

The methylprednisolone protocol. Patients with PCR confirmed COVID-19 who required 4 liters or more of oxygen per minute on admission, or who had esca- lating oxygen requirements from baseline, were recommended to receive IV methyl- prednisolone 0.5 to $1 \mathrm{mg} / \mathrm{kg} /$ day in 2 divided doses for 3 days. Patients who required ICU admission were eligible to extend the IV methylprednisolone course to a maximum of 7 days at the discretion of the medical team. Institutional guidelines also recommended 
hydroxychloroquine $400 \mathrm{mg}$ twice daily for 2 doses on day 1, followed by $200 \mathrm{mg}$ twice daily on days 25 .

Statistical Analysis of clinical data. Survival analysis was performed using the Kaplan-Meier method and log-rank test. More details about the statistical anal-ysis and characteristics of the patient population are included in the Supplementary Materials.

Pathway analysis method. The pathways analysis was performed using the Impact Analysis method 6 , 10,11 . The impact analysis uses two types of evidence: i) the over-representation of differentially expressed (DE) genes in a given pathway and ii) the perturbation of that pathway computed by propagating the measured ex-pression changes across the pathway topology. These aspects are captured by two independent probability values, $p O R A$ and $p A c c$, that are then combined in a unique pathway-specific p-value. More details are provided in the Supplementary Materials and elsewhere 6, 12.

Gene Ontology (GO) analysis method. For each GO term 13, 14, the number of DE genes annotated to the term is compared to the number of DE genes expected just by chance. The $p$-value is computed using the hypergeometric distribution 15, 16 and corrected with FDR and Bonferroni. We also used in intelligent prunning ap- proach inspired by the elim and weight pruning methods 17 . The algorithm constructs a custom cut through the GO hierarchy by starting with the most specific nodes and calculating their $\mathrm{p}$ value with all genes assigned directly to each such node. If a node is significant, it is reported as such. If the node is not significant, the genes associated to the given node are propagated to its direct ancestors and a p-value is calculated for each of those. See Supplementary Materials for full details.

The prediction of upstream Chemicals, Drugs, Toxicants (CDTs) is based on two types of information: i) the enrichment of DE genes from the experiment and ii) a network of interactions from the Advaita Knowledge Base (AKB v1910, www.advaitabio.com). The network is a directed graph in which the source node represents either a chemical substance or compound, a drug, or a toxicant (CDT). We focused our work on FDA-approved drugs that could be repurposed. The edges represent known increase or decrease expression effects that these CDTs have on various genes. The analysis considers the hypothesis that a drug would revert the measured gene expression changes. More details are included in Supplementary Materials.

\section{Conclusions}

Clinical results confirmed the efficacy of the in silico prediction that indicated methyl- prednisolone could improve outcomes in severe COVID-19. These findings are im- portant for any future pandemic regardless of the virus.

\section{Declarations}

Ethics: The clinical study was a multi-center quasi-experimental study at Henry Ford Health System, comprised of five hospitals in southeast and south-central Michigan. The study was approved by the 
institution's Investigational Review Board (\#13739) with waiver of consent.

The authors declare the following competing interests: A patent application has been submitted on $4 / 28 / 2020$ by Wayne State University. This application describes the drug re-purposing method used here. The inventors are Sorin Draghici, Cordelia Ziraldo, Radu Vanciu. A significant part of the analysis was performed with iPathwayGuide which is a software analysis platform similar to Ingenuity Pathway Analysis. iPathwayGuide is commercialized by Advaita Bioinformatics (AdvaitaBio). Sorin Draghici is the founder and CEO of Advaita Bio. Cordelia Ziraldo is an employee of AdvaitaBio. Radu Vanciu was an employee of AdvaitaBio at the time this work was performed. Larry A. Sonna, MD., PhD is the sole proprietor of Bon Air Consulting.

\section{References}

1. Puja Mehta, Daniel F McAuley, Michael Brown, Emilie Sanchez, Rachel S Tatter- sall, and Jessica J Manson. COVID-19: consider cytokine storm syndromes and The Lancet, 2020.

2. Hasan K Siddiqi and Mandeep R COVID-19 illness in native and immuno- suppressed states: a clinical-therapeutic staging proposal. The Journal of Heart and Lung Transplantation, 2020.

3. Janelle Surviving COVID-19: A disease tolerance perspective. Science Advances, April 2020.

4. Daniel Blanco-Melo, Benjamin Nilsson-Payant, Wen-Chun Liu, Rasmus Møller, Maryline Panis, David Sachs, Randy A. Albrecht, and Benjamin R. tenOever. SARS-CoV-2 launches a unique transcriptional signature from in vitro, ex vivo, and in vivo systems. bioRxiv, 2020.

5. Curt M Horvath, George R Stark, lan M Kerr, and James E Darnell. Interactions between STAT and non-STAT proteins in the interferon-stimulated gene factor 3 transcription complex. Molecular and Cellular Biology, 16(12):6957-6964, 1996.

6. Sorin Draghici, Purvesh Khatri, Adi Laurentiu Tarca, Kashayp Amin, Arina Done, Calin Voichita, Constantin Georgescu, and Roberto Romero. A systems biology approach for pathway level analysis. Genome Research, 17(10):1537-1545, 2007.

7. Janice K Louie, Samuel Yang, Meileen Acosta, Cynthia Yen, Michael C Samuel, Robert Schechter, Hugo Guevara, and Timothy M Uyeki. Treatment with neu- raminidase inhibitors for critically ill patients with influenza A (H1N1) Clin- ical Infectious Diseases, 55(9):1198-1204, 2012.

8. Clark D Russell, Jonathan E Millar, and J Kenneth Baillie. Clinical evidence does not support corticosteroid treatment for 2019-nCoV lung The Lancet, 395(10223):473-475, 2020.

9. Chaomin Wu, Xiaoyan Chen, Yanping Cai, Xing Zhou, Sha Xu, Hanping Huang, Li Zhang, Xia Zhou, Chunling Du, Yuye Zhang, et al. Risk factors associated with acute respiratory distress syndrome and death in patients with coronavirus disease 2019 pneumonia in Wuhan, China. JAMA Internal Medicine, 2020.

10. Adi L Tarca, Sorin Draghici, Purvesh Khatri, Sonia S Hassan, Pooja Mittal, Jung- sun Kim, Chong Jai Kim, Juan Pedro Kusanovic, and Roberto Romero. A novel signaling pathway impact analysis. 
Bioinformatics, 25(1):75-82, 2009.

11. Purvesh Khatri, Sorin Draghici, Adi L Tarca, Sonia S Hassan, and Roberto Romero. A system biology approach for the steady-state analysis of gene signal- ing networks. In Progress in Pattern Recognition, Image Analysis and Applica- tions, pages 32-41. Springer, 2007.

12. Adi L Tarca, Sorin Draghici, Purvesh Khatri, Sonia S Hassan, Pooja Mittal, Jung- Sun Kim, Chong J Kim, Juan P Kusanovic, and Roberto Romero. A novel signal- ing pathway impact analysis (SPIA). Bioinformatics, 25(1):75-82, 2009.

13. Michael Ashburner and Suzanna Lewis. On ontologies for biologists: the Gene Ontology-untangling the In 'In Silico' Simulation of Biological Processes: Novartis Foundation Symposium 247, volume 247, pages 66-83. Wiley Online Library, 2002.

14. Gene Ontology Consortium et al. The Gene Ontology (GO) database and infor- matics resource. Nucleic Acids Research, 32(suppl 1):D258-D261, 2004.

15. Sorin Draghici, Purvesh Khatri, Rui P Martins, G Charles Ostermeier, and Stephen A Krawetz. Global functional profiling of gene expression. Genomics, 81(2):98-104, 2003.

16. Sorin Draghici. Statistics and Data Analysis for Microarrays using R and Biocon-ductor. Chapman and Hall/CRC Press, 2011.

17. Adrian Alexa, Jorg Rahnenfuhrer, and Thomas Lengauer. Improved scoring of functional groups from gene expression data by decorrelating GO graph struc- ture. Bioinformatics, 22(13):1600-7, 2006.

\section{Figures}

\begin{tabular}{|c|c|c|c|c|c|c|c|c|c|c|}
\hline \multirow[t]{3}{*}{ Chemical name } & \multirow[t]{3}{*}{$=$} & \multicolumn{2}{|c|}{ COVID19 vs. Healthy } & \multicolumn{2}{|c|}{$\begin{array}{l}\text { lung epithellum (NHBE) } \\
\text { with CoV vs. Control }\end{array}$} & $\begin{array}{l}\text { lung alveolar (A549) with } \\
\text { IAV vs. Control }\end{array}$ & \multicolumn{2}{|c|}{$\begin{array}{l}\text { lung alveolar (A549) with } \\
\text { Cov2 vs. Control }\end{array}$} & \multicolumn{2}{|c|}{$\begin{array}{l}\text { lung alveolar (A549) with } \\
\text { RSV vs. Control }\end{array}$} \\
\hline & & consistent & & consistent & & consistent & consistent & & consistent & \\
\hline & & $\begin{array}{l}(-) / D E \\
\text { targets }\end{array}$ & p-value $=$ & $\begin{array}{l}(-) / D E \\
\text { targets }\end{array}$ & $\mathrm{p}$-value $=$ & $\begin{array}{l}(-) / D E \\
\text { targets }\end{array}$ & $\begin{array}{l}(-) / D E \\
\text { targets }\end{array}$ & p-value $=$ & $\begin{array}{l}(-) / D E \\
\text { targets }\end{array}$ & p-value $=$ \\
\hline Methylprednisolone & & $25 / 27$ & $5.725 \mathrm{e}-10$ & $22 / 22$ & $8.996 e-14$ & & $25 / 26$ & $6.029 \mathrm{e}-14$ & $35 / 37$ & $3.183 e-15$ \\
\hline Gold Sodium Thiomalate & & $22 / 24$ & $5.973 e-8$ & $22 / 22$ & $8.996 e-14$ & & $24 / 25$ & $9.998 \mathrm{e}-14$ & $35 / 36$ & $8.245 e-16$ \\
\hline Prednisolone & & $27 / 34$ & $1.737 e-7$ & $22 / 24$ & $1.373 \mathrm{e}-12$ & & $22 / 23$ & $2.754 \mathrm{e}-13$ & $35 / 41$ & $2.930 e-13$ \\
\hline
\end{tabular}

\section{Figure 1}

The top three drugs proposed for repurposing. The table shows both p-values corrected with Bonferroni, as well as the number of DE genes that would be reverted by each drug. Prednisolone and methylprednisolone are steroids currently used to modulate the immune response in rheumatoid arthritis. The column for A549IAV vs Control is empty because there are no DE genes targeted by these drugs in this contrast. 

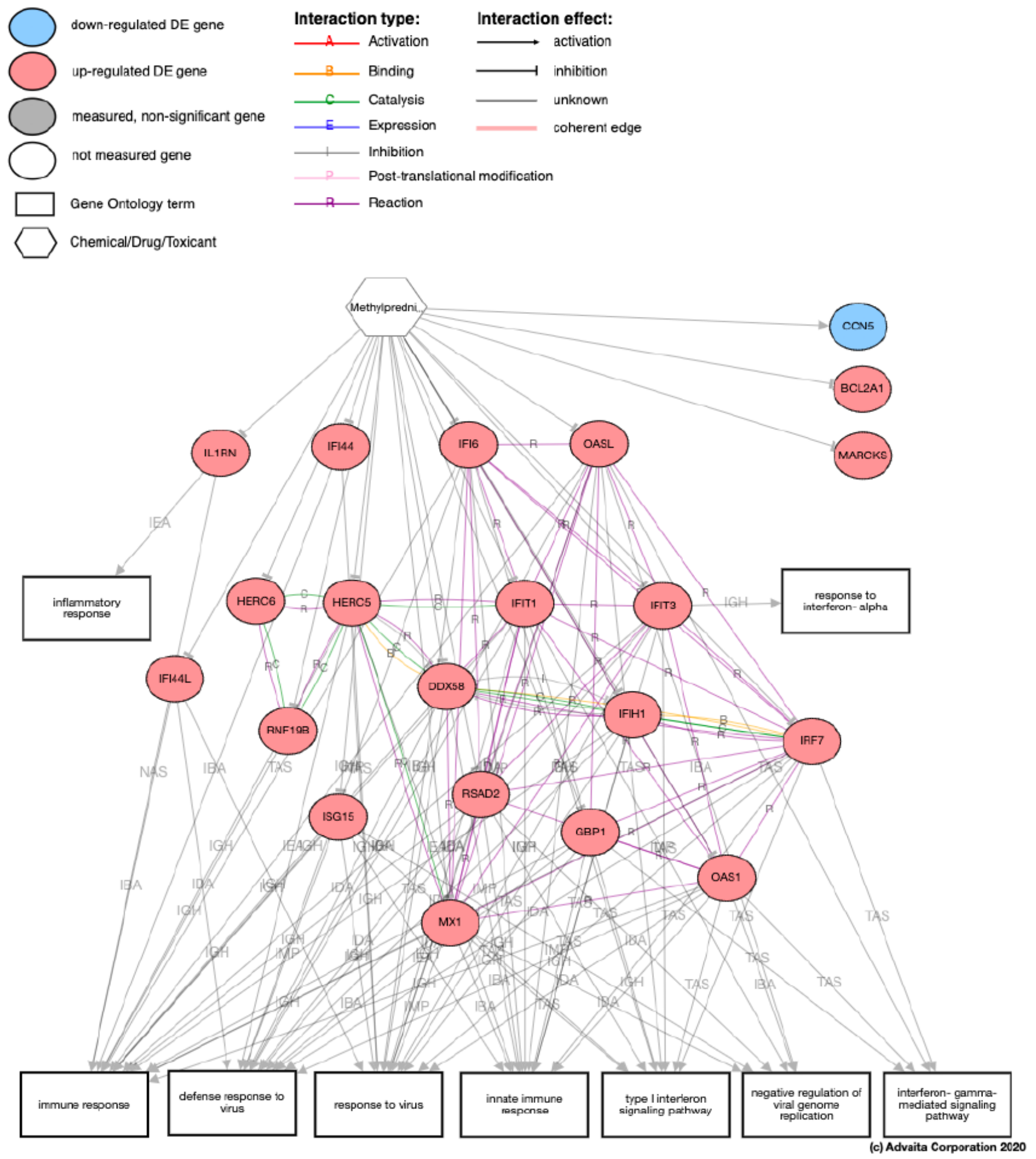

\section{Figure 2}

The putative mechanism through which methylprednisolone act on the genes measured to be DE and how these genes influence the biological processes found to be significantly impacted in the COVID-19 vs Healthy. Note how most of these genes are implicated in one or more of the dys-regulated biological processes. 


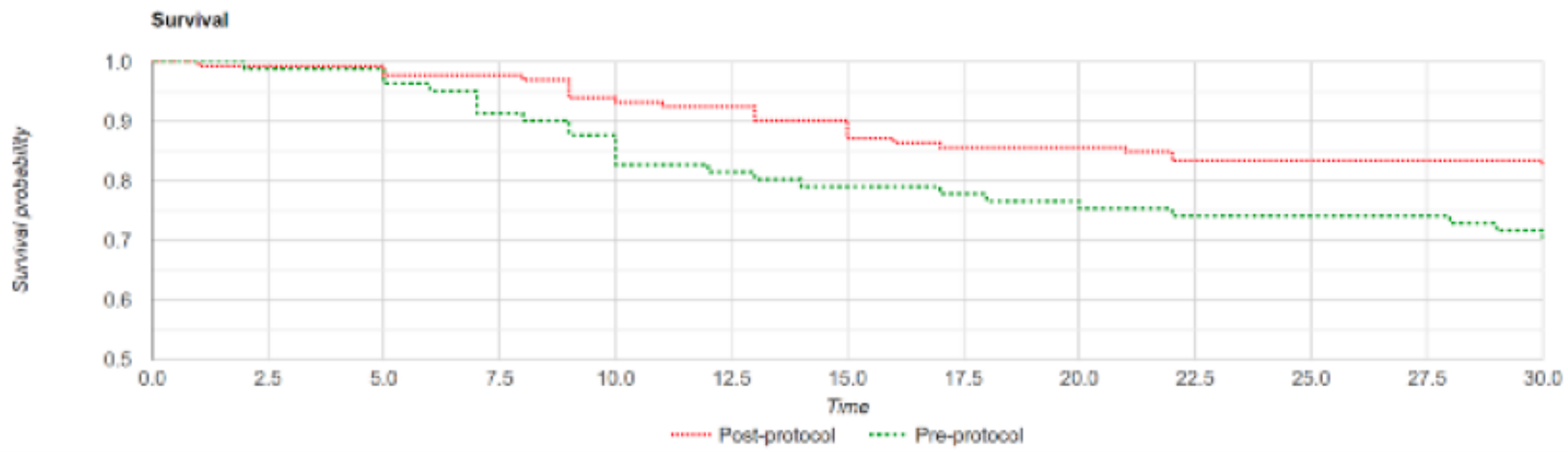

Figure 3

Kaplan Meier survival curve for 30-day mortality demonstrating increased probability of survival at 30days in the post methylprednisolone cohort as compared to the pre-methylprednisolone cohort $(p=$ 0.0204).
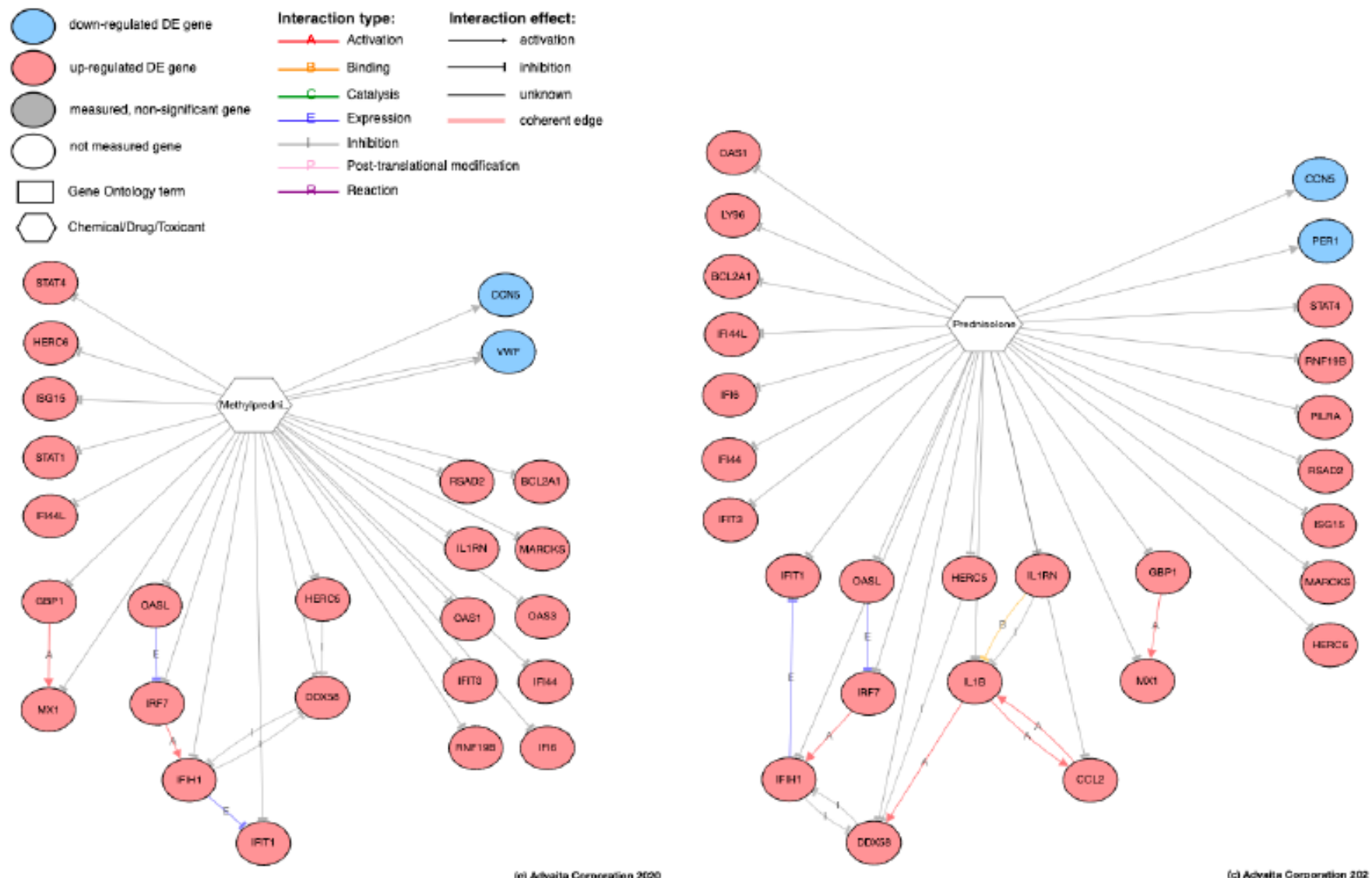

Figure 4 
The putative mechanisms through which Methylprednisolone (left panel) and Prednisolone (right panel) would revert the changes triggered by COVID-19 in the lung tissue.

\section{Supplementary Files}

This is a list of supplementary files associated with this preprint. Click to download.

- Supplementary.pdf 